\title{
EL DETERMINISMO TECNOLÓGICO Y LA "DIALÉCTICA DE LA HISTORIA"*
}

\author{
Amán Rosales Rodríguez \\ Escuela de Filosofía \\ Universidad de Costa Rica \\ arosales@le.ucr.ac.cr
}

RESUMEN: El objetivo de este ensayo es reexaminar el fenómeno conocido como determinismo tecnológico. Si bien la creencia en algo así como un desarrollo tecnológico autónomo, con una especie de lógica propia, ha sido recientemente criticada y descartada por varios filósofos de la tecnología, aquí se piensa que dicha conclusión es injusta y apresurada. Con base en las importantes contribuciones teóricas de Hans Freyer y Friedrich Rapp —usualmente no tomadas en cuenta en la discusión actual—, se muestra cómo se puede ver que el determinismo tecnológico desempeña un papel ineludible en lo que Karl Löwith ha llamado la "dialéctica de la historia".

PALABRAS CLAVE: tecnología, determinismo, historia

SUMMARY: The aim of this paper is to take a second look at the phenomenon known as technological determinism. Although the belief in something like an autonomous, technological development, with a sort of logic of its own, has been recently attacked and dismissed by many philosophers of technology, here it is argued that such a conclusion is hasty and unfair. Relying on the important theoretical contributions of Hans Freyer and Friedrich Rapp — not usually considered in the current debate-, it is showed how technological determinism can be seen to play an ineludible role in what Karl Löwith has named the "dialectic of history".

KEY WORDS: technology, determinism, history

* El autor desea hacer un reconocimiento al DAAD (Servicio Alemán de Intercambio Académico) por el apoyo brindado para la elaboración de este trabajo; así como agradecer al profesor Alois Huning (Universidad de Düsseldorf) y al profesor Friedrich Rapp (Universidad de Dortmund) su estímulo durante la investigación. Importantes recomendaciones y sugerencias fueron planteadas por un árbitro anónimo de Crítica a una versión anterior del ensayo, las cuales han sido incorporadas en su mayoría en el presente trabajo. 


\section{Introducción}

Si bien el presente trabajo no propone, en sentido estricto, una defensa del determinismo tecnológico (o de la autonomía de la tecnología), sino, más modestamente, una reconsideración del tema a la luz de aportes teóricos en apariencia ajenos, algunos de ellos, al debate mismo, incluso así su contenido podría verse con suspicacia. Tan grande ha sido la influencia en los últimos años de los "estudios sociales en ciencia y tecnología", y tan vigoroso, como aparentemente exitoso, ha sido el combate desde sus trincheras a cualquier cosa parecida a fenómenos o procesos "descontextualizados" o socialmente autónomos, que ya se ha convertido en un tópico hablar del fin o la muerte del determinismo tecnológico. Así, en este ensayo se desea argumentar que un problema filosófico cardinal, implícito en la idea del determinismo tecnológico — a saber, la relación entre la autonomía humana y sus obras - no ha sido resuelto, ni tampoco podrá resolverse (en un aspecto importante). Con el respaldo de ideas tanto explícitas como tácitas de un grupo de autores, se arguye que el determinismo tecnológico, en su doble manifestación como efecto y factor corresponsable de lo que Karl Löwith ha llamado, apropiándose creativamente de ciertas ideas de Giambattista Vico, la "dialéctica de la historia", constituye, para escándalo de algunos, un problema que se resiste a ser erradicado sin más del panorama filosófico actual.

El desarrollo argumental se ofrece, en concreto, del siguiente modo: en la sección 2 se proporciona un conjunto de elementos teóricos y definitorios mínimos, con el objeto de preparar mejor el terreno para la exposición en las siguientes secciones. A continuación, en la sección 3 se introduce el enfoque de Löwith, se esboza el contexto de sus ideas, y se enfatiza la relevancia de la noción de la "dialéctica de la historia" para el tema del determinismo tecnológico. Luego, en la sección 4, se presentan las perspectivas de Hans Freyer y Friedrich Rapp, sobre las que también puede decirse que asumen, con sus propios matices y valoraciones, el horizonte interpretativo revelado por Löwith. Finalmente, en la sección 5 se ofrecen comentarios de carácter 
general sobre los argumentos presentados, así como un intento de respuesta a algunos de los problemas analizados.

\section{El determinismo tecnológico en discusión: aspectos preliminares}

Como introducción general al tema y de entre una buena cantidad de opciones teóricas, considérense las siguientes cuatro caracterizaciones o intentos de definición del determinismo tecnológico. Recuérdese que el objetivo es hacer un repaso somero del estado de la cuestión con el propósito de sentar ciertos supuestos básicos como guías para la exposición.

Langdon Winner, en su estudio fundamental sobre el tema, asevera, en procura de una definición, que

en su sentido más fuerte, el determinismo tecnológico depende de dos hipótesis: (1) que la base técnica de una sociedad es la condición fundamental que afecta todos los patrones de existencia social, y (2) que los cambios en la tecnología representan la fuente individual de cambio más importante en la sociedad. (Winner 1977, pp. 75-76)

Uno de los críticos más entusiastas del determinismo tecnológico, Günter Ropohl, sostiene que a pesar de las diferencias parciales entre las distintas concepciones, en el núcleo del determinismo tecnológico se halla "la afirmación de que el desarrollo técnico no depende de factores externos, sino que [éste] determina y domina la situación mental y social de los hombres en tanto que fuerza conductora del cambio social" (Ropohl 1983, p. 86). Por su parte, Peter van Inwagen manifiesta que "el determinismo tecnológico puede concebirse como la idea de que a la luz de la situación pasada (y actual) del desarrollo tecnológico y de las leyes de la naturaleza, el cambio social no puede seguir en el futuro más que un único curso posible" (Bimber 1996, p. 99).

Finalmente, Johan H. van der Pot explica, en términos similares, que, para algunos autores, la

tesis de que en los estados industriales actuales se hace todo lo que es técnicamente posible acabaría en un determinismo tecnológico, 
pues el progreso técnico se da en la sociedad industrial moderna sin influencia de factores externos, en tanto que todo el resto de la cultura se vería reducido a una variable dependiente del progreso técnico. (Van der Pot 1985, p. 714)

Winner tiene razón cuando escribe que muy pocos autores — si es que alguno - estarían dispuestos a suscribir el determinismo tecnológico en sus variantes más fuertes o radicales. No obstante, las descripciones anteriores (incluida la suya propia) son útiles, pues sirven al propósito de conceptuar y sintetizar una serie de intuiciones dispersas que se tienen en torno a dicha noción. Ahora bien, es posible que la expresión "tecnología autónoma", afín a la de determinismo tecnológico, no genere tantas aprehensiones, de no ser porque con ella va aparejada también la idea de que, en el fondo, la autonomía o el supuesto carácter autoalimentado de "La tecnología" (como un todo) no implica, meramente la independencia relativa que caracteriza a sistemas industriales robotizados o automatizados, susceptibles de ser desconectados (al menos en teoría) en cualquier momento, sino su ingobernabilidad final por parte del ser humano. Lo anterior se reconoce tácitamente en las tres modalidades de distinguir o clasificar el determinismo tecnológico que se mencionarán a continuación.

La forma más común de entender el problema es al abrigo de un sentido fuerte o "duro" de determinismo tecnológico. Así, desde tal perspectiva se insiste en dotar a la tecnología y sus productos de un irresistible poder causal propio. Se trata también de un poder coactivo legal que ha sido capturado conceptualmente en la idea, prima hermana del determinismo tecnológico, del "imperativo tecnológico". A partir del enfoque "duro" o fuerte puede pensarse entonces, por ejemplo, que

la continua mejora del ordenador ha seguido una especie de lógica interna [...], por lo que cada "generación" de aumentos de la sofisticación de los ordenadores ha llevado, en una secuencia aparentemente predeterminada, a la siguiente. (Smith y Marx 1996, p. 13) 
A Smith y Marx se les debe la distinción entre un tipo "duro" y otro "blando" de determinismo. Este último, contrariamente al primero, pese a reconocer la influencia colosal de la tecnología en todo ámbito social, rechaza la conveniencia de "concebir la “tecnología' per se como el agente causal histórico", y propone situar su desarrollo "en una matriz social, económica y política y cultural mucho más variada y compleja” (p. 15).

La distinción citada entre una variante "dura" y otra "blanda" de determinismo tecnológico se corresponde bastante bien con el primer y el segundo enfoques de la clasificación propuesta a su vez por Ilkka Niiniluoto, quien distingue entre una perspectiva "romántica" y otra "tecnocrática" de concebir la tecnología. La primera tiende a personalizar o reificar la tecnología hasta convertirla en una especie de entidad maligna, no sólo independiente del ser humano, sino dotada de iniciativa propia. La segunda nace de la aceptación, más o menos resignada, de que la racionalidad tecnológica y sus productos, en tanto que factores dominantes en las sociedades industriales, deben imponer los valores y criterios de funcionamiento ante los que debe rendirse, por su propio bien, la dinámica social. Mientras que Jacques Ellul sería, de acuerdo con Niiniluoto, el autor representativo de la visión "romántica", Helmuth Schelsky y su teoría del "Estado técnico" lo serían de la "tecnocrática". Entretanto, la taxonomía propuesta por Bruce Bimber sigue un derrotero similar al sugerido por Marx, Smith y Niiniluoto. En opinión de Bimber, pueden reconocerse una variante "nomológica" (en general equivalente a los anteriores tipos "duro" y "romántico") y otra "normativa" (correspondiente a los tipos "blando" y "tecnocrático") de visualizar el determinismo tecnológico. En tanto que, de acuerdo con el punto de vista "normativo", "es precisamente lo que los individuos han llegado a pensar y desear lo que ha dado lugar a la sociedad tecnológica", desde la posición "nomológica" se enfatiza que "la sociedad dirigida por la tecnología surge independientemente de los deseos y valores del hombre" (Bimber 1996, pp. 98-100).

En las tres propuestas anteriores de clasificación llama la atención el hecho de que el fenómeno del determinismo tecnológico se entienda desde una visión "bipolar" que tiende a excluir, 
según el caso, a su contrapartida. En ese sentido, resulta tentador plantear que ha sido justamente a consecuencia de tal separación y rivalidad entre posturas "duras" y "blandas" — con sus respectivas variantes según cada autor- que no se ha considerado el modo en que la idea del determinismo tecnológico apunta, necesariamente, hacia un estado más amplio de cosas, un estado que involucra la tensa y complicada relación de los sujetos humanos con su propia historia. Se trata de una relación en la que ambos elementos son, en gran medida, autónomos y dependientes al mismo tiempo de los imperativos del otro.

La idea de que con el desarrollo tecnológico va implícita una especie de fuerza normativa que demanda su realización imperiosa o incondicional, no sólo ha sido considerada coherente por muchos, sino que también ha sido aceptada por no pocos reconocidos autores. Específicamente en las filas de la llamada "crítica cultural alemana" (Kulturkritik), las reacciones de los intelectuales "conservadores" (de Burckhardt a Spengler, de Sombart a Jaspers, etc.) ante la amenaza deshumanizante de "La máquina" están bien documentadas. Su importancia descansa sobre todo, para el caso del presente trabajo, en un aspecto particular: con la perspectiva crítico-cultural se comienza a examinar — de modo gradual - el determinismo del sistema tecnológico-industrial en su conjunto, y ya no simplemente el de los medios técnicoinstrumentales: máquinas, herramientas, equipos, etc. Este punto resulta llamativo, como habrá que ver con mayor detalle, en un autor como Hans Freyer, testigo de una época en Alemania marcada por la ambivalencia hacia el desarrollo tecnológico.

Lo antedicho respecto de la crítica cultural alemana todavía merece destacarse, por cuanto la perspectiva común de los tres autores principales que se discutirán más adelante (Löwith, Freyer y Rapp) tiende a coincidir con ella en el sentido de afirmar en efecto que, instrumentalmente, no hay mucho que sostenga la idea de una pila de medios tecnológicos convertida totalmente en autónoma. El que ciertos objetos y sistemas tecnológicos, como el teléfono a comienzos del siglo xx o la internet en la actualidad, tengan usos no previstos por sus diseñadores originales, tampoco resulta especialmente sorpresivo. Salvo, entonces, que se caiga en la tentación de hipostasiar la suma de medios materiales para 
el dominio y la transformación de la naturaleza, no parece haber justificación alguna para aceptar la idea de que "La tecnología" o su "Esencia" (recuérdese a Heidegger) es soberana en el mundo actual. Así, una concentración excesiva en el objeto técnico o tecnológico fácilmente puede conducir a lo que William Leiss ha llamado la "hipérbole tecnológica", esto es, "la exageración sistemática e ilegítima de los efectos de los aspectos puramente técnicos de las tecnologías sobre los sistemas sociales" (Leiss 1990, p. 34).

Aún hay que resaltar, a propósito del comentario anterior, cómo las observaciones de Leiss ya revelan, con bastante nitidez, cierta actitud ambigua ante la autonomía de la tecnología. Una actitud cuyos orígenes hay que localizar en el contexto más amplio, histórico, del determinismo tecnológico. Pues si bien, por una parte, Leiss parece coincidir con los críticos del determinismo tecnológico al indicar que su propio punto de vista "considera las tecnologías como mixturas de técnicas (techniques) y respuestas sociales". Por otra, en el capítulo conclusivo de su obra, se muestra más comprensivo de las "fantasías" en torno a una tecnología autónoma, en la medida en que tal noción representa una "metáfora acerca de la paradoja del control con respecto a la relación entre la humanidad y la naturaleza". Con la "paradoja del control", Leiss sugiere una idea que está muy cerca, como pronto se verá, de lo que está en liza con la propia "dialéctica de la historia" de Karl Löwith (Leiss 1990, pp. 35, 141).

\section{Karl Löwith y la "dialéctica de la historia"}

De la vasta producción de Karl Löwith como historiador y filósofo sobresale, sin duda, Meaning in History, su importante obra de 1949. Sin embargo, no menos importantes para los propósitos de este trabajo son sus posteriores ensayos de 1950 y 1963. Recuérdese que lo que interesa subrayar es, ante todo, de qué modo las reflexiones de Löwith acerca del culto occidental a la historia, secuela característica del proceso de secularización o naturalización sufrido por la concepción histórica cristiana, tienen consecuencias - indirectas, sí, pero no por ello menos 
claras - para una reconsideración del determinismo tecnológico. Previamente a un desarrollo más explícito de dicha idea, se debe bosquejar la base del enfoque histórico filosófico de Löwith. El esbozo se realizará: en primer lugar, en 3.1, y habrá que seguir la pista a la interpretación que él hace, extrayendo sus propias conclusiones, de los sistemas históricos de Comte y Vico. Asimismo, en segundo lugar, en 3.2 - pero mucho más sintéticamentehabrá que destacar la crítica de Löwith a ciertos enfoques, como los de Hegel y Marx, que también marcaron la pauta, junto con el de Comte, para un proceso combinado de absolutización de la historia y de separación radical, de aliento nihilista, entre la propia historia y la naturaleza.

3.1. Respetando el orden establecido por Löwith (1949), en el sentido de estudiar primero a los autores más recientes, ha de tenerse en cuenta la concepción histórica comteana. Nótese que Löwith busca identificar la influencia de la interpretación cristiana de la historia incluso en aquellas perspectivas que más parecen contradecirla. Es más, es en estas últimas donde él detecta su mayor impacto cultural. La secularización de dicha visión de mundo se muestra entonces de modo característico en el sistema positivo de Comte, único sistema que a criterio de Löwith puede rivalizar con el de Hegel en cuanto a amplitud (aunque no en profundidad). Para el autor francés, sin embargo, ya no es el individuo y su salvación personal, como en el cristianismo, lo que debe acaparar la atención, sino el destino colectivo de la humanidad. Reacio a claudicar en su "optimismo evolucionista", Comte no pudo ver, según lo entiende Löwith, que todo progreso en el dominio humano sobre el mundo acarrea necesariamente un retroceso, pérdidas en otros aspectos. El gran positivista nunca se percató de que "todos los medios de progresión son igualmente, en tanto el hombre mortal esté implicado en el proceso histórico, otros tantos medios de regresión" (p. 89). La perspectiva histórica adoptada por Comte, prosigue Löwith, no ofrece más que una gigantesca simplificación, con todo y lo bien intencionada que pueda ser, de las complejidades, reconocidas por la propia visión cristiana, del desarrollo histórico concreto. 
La visión positivista pinta entonces, según Löwith, un cuadro distorsionado, falso de una realidad en la que siempre habrá lugar no sólo para los éxitos, sino también, e incluso más, para todo tipo de catástrofes, pérdidas y regresiones:

Mientras que la visión griega de la historia, así como la cristiana, estuvo abierta a los severos hechos de la hybris y la nemesis, del orgullo y la condena, la perspectiva positivista de la historia no puede sino falsificar la realidad histórica en aras de una inalcanzable solución secular. (p. 89)

Frente a la ingenua y "unidimensional interpretación" comteana, Löwith urge a que se acepte el hecho de la cruda evolución histórica, que no puede ofrecer el consuelo de la marcha lineal y completamente progresiva imaginada por Comte. La "inmensa realidad de la historia", que presenta rasgos tanto humanos como "inhumanos", se resiste a ser encajada en el molde simplista del progreso ilimitado y constante, siempre benéfico para el ser humano. Es que, para Löwith, aquella realidad

es al menos tan rica en contradicciones como la Natur de Goethe o el "mundo dionisiaco" de Nietzsche, y el poderoso río de la historia que rompe el dique e inunda un país es esencialmente la misma corriente pacífica que parece disfrutar de sus ordenadas riberas. (pp. 89-90)

Löwith descubre una visión más acorde con la historia real en autores anteriores a Comte, como por ejemplo Turgot, y sobre todo Vico y su Scienza Nuova. A diferencia de su posterior coterráneo positivista, Turgot, en el curso de su laboriosa tarea de sustituir la providencia sobrenatural por la ley universal del progreso, se dio cuenta de "que los movimientos históricos no son una simple progresión unidimensional, sino un intrincado sistema de apasionadas intenciones y resultados inesperados" (Löwith 1949, p. 102). Dicha intuición de Turgot sobre el carácter complejo de la evolución histórica, alcanza su culminación en la noción fundamental de la "dialéctica de la historia", extraída de Vico por Löwith, y adaptada o enriquecida, como se verá más adelante, por Freyer y Rapp. 
Tal vez fue Vico, de acuerdo con la interpretación de Löwith, el primer autor que percibió con claridad el carácter dialéctico de la historia humana. Ésta adopta la forma de un proceso sometido de modo constante al vaivén de fuerzas, intenciones e impulsos, individuales y colectivos, por lo general también contrarios y conflictivos entre sí. De ese modo, el mundo histórico fue captado por Vico como "un mundo hecho por hombres $y$, al mismo tiempo, sobrepasado por doquier por algo que es más cercano al destino que a la libre elección y acción" (p. 126, las cursivas son mías). Así, la "dialéctica de la historia" representa el modo característico en que se ofrece, en su totalidad, la evolución cultural humana. La "solución" que ofrece tal choque dialéctico de intenciones y realizaciones para el problema del sentido de la historia, se asienta en una reinterpretación del papel de los actos humanos. Éstos los entiende Vico — en la explicación de Löwith - como una suma de eventos cuya justificación final apunta más allá de los deseos y proyectos particulares. Tal suma ofrece testimonio, en efecto, de un "Plan divino". Este "Plan", sin embargo, tampoco puede ejecutarse directamente haciendo caso omiso del carácter fortuito o contingente de la historia humana. Esta historia o "mondo civile", en cuanto tal, aparece más bien como un juego "histórico-cíclico" sucesivo e indefinido de cursos (corsi) y recurrencias (ricorsi). El progreso, desde el enfoque de Vico, no es más que una sucesión de etapas "sin fin y realización" última.

No hay entonces, en Vico, asegura Löwith, una concepción ingenuamente lineal del progreso histórico. "Enraizada" hasta cierto punto en el ámbito contingente e imperfecto de la acción humana, la providencia actúa de forma más bien inmanente, con la ayuda, por ejemplo, de "las costumbres naturales del hombre". Estas costumbres, arguye Vico, persiguiendo muchas veces sus propios, estrechos intereses y motivos, "sirven a fines más amplios", destinados a "conservar la raza humana sobre la Tierra". En su "inmensurable bondad", la Divina Providencia debe, sin embargo, ordenarlo todo "hacia un bien siempre supe- 
rior a aquel que los hombres se han propuesto a sí mismos" (Sullivan 1970, p. 13). ${ }^{1}$

3.2. Löwith vislumbra en un modelo histórico heredero sobre todo de Hegel y Marx, los antecedentes decimonónicos de la actual comprensión dinámica de la historia, obsesionada por la idea del progreso y el imperio que ésta extiende - con los medios de la ciencia y la tecnología - sobre la naturaleza. La concepción quiliástica de la historia encuentra su exposición definitiva en el intento hegeliano por armonizar, mediante "el ardid de la razón", la contingencia histórica trivial con los más altos designios, no enteramente manifiestos, de la Divinidad. Dicho "ardid" es en efecto para Hegel, según Löwith, nada menos que "una expresión racional de la Divina Providencia" (p. 56). En

1 No sería correcto seguir adelante sin hacer notar, por lo menos de paso, la afinidad de ese enfoque derivado de Vico con ideas posteriores importantes, como la de las "unintended consequences" del autor escocés del siglo XVIII, Adam Ferguson, así como con la "ungesellige Geselligkeit" kantiana, y la del "List der Vernunft" hegeliano. Entre otras cosas, en las tres ideas es común el acento puesto sobre el carácter complejo de la realidad histórica, en el sentido de que en ella tienen lugar, de modo más o menos armónico, tanto acciones individuales, racionalmente planificadas y ejecutadas, como eventos o acontecimientos que no parecen responder a designio consciente o plan racional alguno. Así, en Kant, la "insociable sociabilidad" (el antagonismo o la discordia entre los seres humanos) sirve en última instancia, de forma inconsciente, al elevado propósito de levantar un orden social legaliforme (cfr. Kant 1987, p. 8). En Hegel, "la idea universal" no puede aceptar mezclarse directamente con lo contingentemente determinado. Para cumplir sus objetivos se vale del "ardid de la razón", éste consiste en hacer "que las pasiones obren por ella", sin importar si en el proceso lo particular es sacrificado o abandonado a lo universal: "La idea no paga por sí el tributo de la existencia y de la caducidad; págalo con las pasiones de los individuos" (Hegel 1999, p. 97; cfr., además, Löwith 1949, pp. 52-59). Finalmente, aunque en realidad previa a las propuestas anteriores, debe mencionarse la teoría de las "consecuencias no intencionadas", "no deliberadas" o "involuntarias" de Ferguson (compartida con variaciones por otros autores más conocidos de la llamada Ilustración escocesa, como David Hume y Adam Smith). Su núcleo está concentrado en un célebre pasaje de su $A n$ Essay on the History of Civil Society de 1767 (Ferguson 1995, p. 119). En esta obra, su autor señala cómo "las naciones topan (stumble) con instituciones (establishments) que son por cierto el resultado de la acción humana, pero no la ejecución de un designio humano". En otra oportunidad se espera explorar más a fondo la conexión entre la teoría de las consecuencias no intencionadas - desarrollada al máximo, como 'caso específico" de la "dialéctica de la historia", durante el siglo XVIII-, con otros problemas vinculados a la dinámica del desarrollo tecnológico y sus implicaciones deterministas más recientes. 
relación con Marx, Löwith asegura que su materialismo histórico sigue orbitando alrededor del mismo punto de vista escatológico y profético (el proletariado será el encargado de finalizar la historia) activo en la filosofía hegeliana de la historia. Es que en Marx aún se mantiene, según Löwith, la misma tensión entre trascendencia y contingencia que Hegel más bien procuró resolver con su fórmula de la racionalidad de lo real y viceversa. En ese sentido Löwith puede decir: "Comparado con Marx, el realista más grande es Hegel" (p. 51). Löwith colige de la tendencia europea moderna (con sus raíces hebreo-cristianas) hacia la absolutización del mundo histórico, una serie de consecuencias significativas para el nudo de relaciones entre historia, tecnología y naturaleza. Entre ellas se podrían contar las dos siguientes ( $c f r$. Löwith 1950 y 1963).

Por un lado, la primera consecuencia se manifiesta en la dualidad entre historia y naturaleza que hasta hoy en día se conserva en Occidente. En ese sentido, la separación de los dos ámbitos extiende su influencia hasta la filosofía "existencial" heideggeriana y su consideración de un "Dasein" histórico y "mundano", contrapuesto a una esfera de entes carentes por completo de historicidad y comprensión de su estatus ontológico. El punto de vista del autor de Sein und Zeit ofrece testimonio, según Löwith, de la influencia combinada del dualismo cartesiano con la propia escisión establecida por Vico entre el mundo histórico y el natural, a pesar de esfuerzos monistas como los de Hegel y el contenido en la Naturphilosophie de Schelling. De ahí sólo hay un paso para creer que la naturaleza constituye una mera reserva (el "Bestand" de Heidegger) que aguarda por su aprovechamiento tecnológico. En todo esto, Löwith percibe la fase culminante del nihilismo occidental: la "historicidad" no logra integrar en una, la realidad humana y la natural, sino que agrava el sentido de desamparo (Heimatlosigkeit) típico de la modernidad. Es que el mundo natural moderno no es más un cosmos autosuficiente y creativo, sino, ante el acoso permanente de lo humano y su tecnología, "un mundo desnaturalizado" (Löwith 1950, p. 293). Aquí hay en efecto, por lo menos en el diagnóstico de algunos problemas, una triple coincidencia entre Löwith, ciertas tesis de la crítica cultural y su ex maestro Heidegger. Para este último, 
la decadencia metafísica de Occidente se revela, entre otras cosas, en la conversión de la naturaleza a un vulgar "recurso" que sólo puede ser experimentado - siguiendo intuiciones de Ernst Jünger en su Der Arbeiter (1932)__, "a la luz del proyecto nietzscheano del ente como voluntad de poder" (Jünger y Heidegger 1994, p. 80). ${ }^{2}$

Por otro lado, la obsesión moderna por la historia ha comportado también nada menos que "la fatalidad del progreso", como lo ha descrito elocuentemente el propio Löwith ( $c f r$. su conocido ensayo de 1963). Es que el progreso histórico se da en la forma de un proceso de apropiación creciente de la naturaleza mediante la cultura; pero, mientras que la perspectiva "clásica" del progreso (Löwith cita a Lucrecio) mantenía a la vista la precariedad de los logros "progresistas" humanos, la moderna, de ascendencia cristiana, dirige osada y "revolucionariamente" la mirada hacia el futuro. Con la incorporación plena de la ciencia y la técnica en los sistemas industriales, el progreso termina por absorber todo proyecto histórico. La sed de avances se vuelve insaciable y desmesurada. Ésa es la perspectiva que ha dominado en el hemisferio occidental hasta por lo menos la Primera Guerra Mundial, cuando la idolatría del progreso sufrió daños formidables aunque no irreparables. De momento, Löwith constata que la ambivalencia, como estado de ánimo preponderante, es la secuela final de siglos de incubación, desarrollo y decadencia de la hasta hace poco reinante mentalidad progresista, quiliástica occidental. Es en esta situación actual de acciones históricas —es decir, de origen humano, pero no en absoluto de designio humano bien

${ }^{2}$ Un brillante tratamiento de este mismo complejo de problemas lo proporciona Hans Jonas (otro ex discípulo de Heidegger), quien cita de pasada a Löwith en el curso de su propia crítica al nihilismo existencialista, véase Jonas 1966, p. 216, nota 7. La relación Nietzsche-Heidegger-Jünger constituye, desde luego, un vasto campo temático por derecho propio. Se ofrecen más elementos aclaratorios en Rosales 1994. Más recientemente, Eric Jakob ha insistido en la influencia de Jünger sobre lo que puede estimarse como una interpretación quiliástica de la técnica, hecha por el Heidegger antimodernista de los años treinta y cuarenta del siglo pasado ( $c f r$. Jakob 1996, p. 42 et seq.); Karl Löwith expone críticamente sus reparos a la filosofía heideggeriana en su incisivo estudio Heidegger —Denker in dürftiger Zeit (1953). 
definido - múltiples y complejas, donde la consideración del determinismo tecnológico adquiere relevancia analítica como una figura en la que se pone de relieve con claridad, la paradójica relación, peligrosamente real, de la modernidad con los frutos de su propia historia.

Es tiempo de volver a la interpretación que Löwith realiza de Vico, y al nexo más directo que cabe establecer, a partir de ahí, con el tema de este trabajo. En su presentación de lo que él mismo llama la "teología civil racional" de Vico, salta a la vista la preferencia de Löwith por un enfoque complejo de la realidad histórica, muy cercano en efecto a la propuesta por el autor italiano. Se trata de un enfoque, el de Vico, tal que, al convertir la trascendente Divina Providencia en una inmanente "ley de la historia", ha revelado la ambigüedad misma del proceso histórico, en la medida en que éste ha sido dejado ahora a sus propios e inciertos recursos puramente humanos. Como Löwith lo señala un tanto resignadamente, con la irrupción de la ciencia moderna "ahora estamos, como nunca antes, 'haciendo historia', y sin embargo nos encontramos abrumados por ello debido a que la historia se ha emancipado de sus límites clásicos y cristianos" (1949, p. 194). El acento ya indicado sobre el juego permanente de avances y retrocesos, ganancias y pérdidas, típico de la "dialéctica de la historia", podría extenderse, en la actualidad, a lo que cabe llamar la dialéctica del desarrollo tecnológico. La idea, parafraseando a Löwith, rezaría así: ahora estamos como nunca produciendo tecnología, y sin embargo nos encontramos abrumados por ello debido a que la tecnología (pero aquí tal vez léase mejor: el conjunto de actos humanos mediados por la tecnología) tiende siempre, "por su misma naturaleza", a traspasar todo límite una vez que éste es alcanzado. La tecnología no es una instancia suprahistórica, desencarnada del mondo civile; pero, en tanto que motor por antonomasia del progreso material en la edad moderna, su poder de determinación (para bien o para mal), sí supera con frecuencia todo planeamiento y expectativa originales. 


\section{Perspectivas complementarias: Hans Freyer y Friedrich Rapp}

El calificativo de "complementarias" indica ya que los enfoques de Freyer y Rapp no deben ser entendidos como simples apéndices del de Löwith. Se introducen aquí con la intención de reforzar la propuesta insinuada anteriormente; a saber, la necesidad de situar el tema del determinismo tecnológico en el contexto de una discusión más amplia en torno a la relación fundamental, de interacción causal mutua, a dos vías, entre los sujetos humanos y su historia.

La relevancia del aporte de Hans Freyer para la discusión en torno al determinismo tecnológico no ha sido suficientemente reconocida. Si bien un autor como Ulrich Teusch, más recientemente, y el propio Rapp, desde hace muchos años, han adaptado algunas de sus ideas, todavía hay mucho que recuperar de sus escritos para las discusiones actuales. Aunque es exacto afirmar que en los trabajos de Freyer aparece la pregunta constante de la filosofía de la técnica "acerca del papel del ser humano en la modernidad y de las posibilidades que le quedan para conducir, ya no solamente el progreso técnico, sino del todo la historia" (Huisinga 1996, p. 24). Lo cierto es que al exponer la posición de Freyer, simplemente, como un ejemplo más de cierta postura teórica (como lo hace Huisinga, quien cita también entre otros a Ellul y Arnold Gehlen), tiende a restársele originalidad a su enfoque. ¡Efectivamente: la importante vinculación que con buen tino establece Huisinga entre el ámbito específico de la acción tecnológica y el más general de la historia no parece haber sido una cuestión de interés permanente en la filosofía de la técnica! Desde esta perspectiva es posible insistir en la originalidad de las aportaciones teóricas combinadas de Löwith, Freyer y Rapp. De hecho, estos aportes pueden ser también compatibles (en algunos aspectos) con los provenientes de enfoques relativamente más nuevos, de acento "evolucionista" sobre el desarrollo tecnológico. ${ }^{3}$

3 Se piensa sobre todo en el conocido enfoque auspiciado por George Basalla (1988). Este autor formula una interesante teoría de la evolución tecnológica, basada en la interacción de cuatro conceptos: diversidad, continuidad, novedad y selección, que él mismo encuentra compatible con la idea de una 
4.1. Para la presentación, necesariamente muy sintética de las ideas de Freyer, se tomarán en cuenta algunas pistas contenidas en las que son, muy posiblemente, sus obras más importantes del periodo de posguerra, correspondientes a los años 1955 — obra que se citará según la versión castellana de 1966y 1965. De entrada, el diagnóstico cultural de Freyer parece coincidir con el de la desencantada crítica cultural alemana respecto del fenómeno técnico y, más recientemente, del imperativo tecnológico. Por ejemplo: "El hombre ya no le pregunta al instrumento si es apropiado, el instrumento pregunta al hombre si no quiere hacerlo funcionar, pues puede hacerlo" (1966, p. 175). Aunque otros numerosos textos pueden acumularse en ese sentido, lo cierto es que la perspectiva teórica de Freyer sólo superficialmente puede, sin más, ser catalogada de favorable al determinismo tecnológico. Para Freyer, la técnica, y en especial la moderna o tecnología, con todo y que representa "el símbolo y la concentrada esencia de la época actual" (p. 176), también debe ser incluida dentro de la propia "dialéctica de la historia" ya presentada en relación con Vico y Löwith. Lo anterior puede mostrarse sobre la base de dos conceptos-guía tomados de las obras de Freyer en consideración: las nociones de progreso, entendido como "progreso real" (progrès réel, término que Freyer retoma de Georges Sorel), y sistema secundario.

tecnología autónoma (véase la p. 205, donde se cita explícitamente la versión determinista de Winner). Basalla bautiza su posición de "determinismo modificado o atenuado", por cuanto afirma que los sujetos tropiezan con condiciones que restringen considerable, pero no totalmente, su capacidad de selección de ciertas tecnologías ( $c f r$. pp. 205-206). Hasta aquí podría haber coincidencia con pareceres afines por encontrar en Freyer y Rapp. Empero, hay también algunas diferencias entre la perspectiva determinista de Basalla y la centrada en la "dialéctica de la historia" que vale la pena, mencionar por lo menos brevemente. De momento basten dos: 1) La concentración del enfoque de Basalla en la proliferación de los aparatos técnicos, mientras que la otra lo hace en ciertos procesos históricos, de amplio impacto social que también incluyen a la tecnología (como la industrialización). 2) Desde la segunda posición es importante considerar la noción de "progreso tecnológico" en su imbricación con otros factores: sociales, económicos, políticos, etc. Basalla aboga más bien por su independencia respecto de estos últimos ( $c f r$. p. 216). Por desgracia, no es posible ofrecer aquí una comparación más elaborada (y justa). 
Ante todo, Freyer llama la atención sobre un rasgo definitorio por excelencia de la época contemporánea y su carácter "progresista". Es el hecho de que esta época se encontraba en un momento de transición constituido por el "umbral industrial" (industrielle Schwelle), que a su vez se caracteriza por impulsar, según él, un modo hasta ahora extraordinario de organización general del trabajo y la vida. Al "progreso real" o concreto, producto del actual "umbral" histórico y experimentado en procesos igualmente novísimos de industrialización y tecnificación, hay que concederle que produce una suerte de coacción objetiva (Sachzwang) que, si bien carente en sí misma de la fuerza del "deber ser", actúa en la práctica "con la fuerza normativa de lo fáctico" ( $c f r$. Freyer 1965, pp. 301, 302, 308). El "progreso real" industrial del presente casi adquiere, en la descripción de Freyer, la fuerza de un suceso natural que tiende a extenderse incontenible por todo el planeta. Según Freyer, la presencia de "coacciones objetivas" (Sachzwänge) señala a un fenómeno eminentemente moderno, consecuencia del crecimiento gradual pero seguro de la "cultura industrial".

Dicha cultura, movida con el impulso otorgado por el progreso tecnológico, presenta ciertos rasgos distintivos que articulan, en opinión de Freyer, su propio carácter "coactivo" sobre personas e instituciones (véase, para lo que sigue, Teusch 1993, pp. 281-282, y Freyer 1965, pp. 324-325): a) El proceso tecnológico-industrial no tiene, de cara al porvenir, una meta definida, simplemente "avanza" confiado hacia delante. b) Sobre su curso futuro general no es posible ofrecer una prognosis confiable a largo plazo. c) Aunque inabarcable en su conjunto, el proceso coactivo global sí permite un análisis, siquiera imperfecto o parcial, a partir de sus manifestaciones parciales o segmentos constitutivos de progreso (esto es, la "coacción objetiva" no excluye cierta planificación sectorial). d) Pero son también esas mismas manifestaciones de progreso en parcelas particulares (sobre todo de naturaleza científica-tecnológica), las que facilitan la expansión mundial acelerada del progreso como 
totalidad, y por ende su penetración en todo ámbito de la vida social. ${ }^{4}$

En la medida en que la opción del "progreso real" - junto con la de su concomitante desarrollo científico, técnico e industrialparece haber sido asumida nolens volens por Occidente, Freyer procura identificar ciertos ingredientes típicos de este hemisferio que propiciaron su aparición y desenvolvimiento futuro. En la combinación de tres factores: la ciencia, la técnica y el ethos burgués capitalista respecto del trabajo, Freyer cree descubrir la matriz de la "cultura industrial" (Industriekultur) del presente. Esta forma cultural, dominante en el presente, se encuentra determinada por las normas técnicas "de la eficiencia pura, la utilidad máxima, del one best way” (Freyer 1965, p. 186. Aquí se divisa la influencia de La Technique ou l'enjeu du siècle, de Ellul). El mundo contemporáneo se identifica, en el diagnóstico de Freyer, con la época en que las expresiones más claras del determinismo histórico, los llamados por él "sistemas secundarios" de la producción masiva, de los medios de información, transporte, y del manejo o administración eficiente de recursos, materiales y humanos, proyectan su radio de influencia en todo ámbito de vida, individual y social. Tales sistemas son un subproducto del proceso irrefrenable de industrialización optado por Occidente, un proceso que "se mantiene en marcha a sí mismo y se impele hacia delante al modo de una reacción en cadena" (Freyer 1966, p. 229). Son "secundarios" no únicamente en la medida en que son artificiales y representan, como bien lo aclara Jerry Z. Muller, "una alineación de un orden más primario y orgánico social", de mayor "continuidad con la naturaleza y el pasado" (1987, p. 339), sino que lo son ante todo por haber aceptado, en sustitución del orden primariamente conservador y

4 De paso merece destacarse que la creencia en ciertas "coacciones objetivas", dominantes sobre la situación social y espiritual de los seres humanos, tuvo un fecundo desarrollo no sólo en Freyer, sino además en sus no menos famosos ex discípulos o asociados, Arnold Gehlen y, sobre todo, Helmuth Schelsky. La discusión llega incluso hasta Habermas y su polémica en contra de la tecnocracia. En lo tocante a estas cuestiones, el estudio fundamental de Wilhelm Glaser (1972) no ha perdido vigencia. 
estabilizador de la cultura, el orden "progresista" de inspiración técnica racional.

En ese sentido, del "sistema secundario" se desprende una "ley estructural" que marca el paso de cuatro grandes tendencias características, según Freyer (1966), del "umbral industrial" contemporáneo. Nótese que tales tendencias (o posibilidades del hacer) se ofrecen al modo de componentes de un ambiciosísimo plan técnico universal de intervención sobre la realidad y la historia. Se trata de las posibilidades de "fabricar objetos", "organizar el trabajo", "civilizar al hombre" y "consumar la historia". Los "sistemas secundarios", tomados en su conjunto, incluyen entonces, en la época actual, no sólo instrumentos y objetos, sino sobre todo, formas eficazmente complejas de organización del trabajo y la producción. Permiten la administración eficiente de los recursos, pero exigen como precio la inclusión del individuo, su adaptación como pieza funcional, y quizá también intercambiable, dentro de una estructura productiva que parece trabajar autónomamente. Para Freyer, la "cultura industrial" del presente personifica el "sistema secundario" por excelencia ("este sistema sobrio y objetivo de linaje occidental", 1965, p. 165).

En realidad, acerca de la teoría de los "sistemas secundarios" y del "espíritu técnico" en ellos activo, puede ser válido generalizar la opinión que el propio Freyer emite a propósito de la teoría marxista de la revolución proletaria. Esta última noción se montaría a horcajadas entre el voluntarismo individual (por medio de la acción violenta de la clase trabajadora) y el determinismo histórico (en cuanto se tiende a pensar de ella como un inevitable proceso natural): "Este estar uno al lado del otro del determinismo y la acción programática se halla más o menos en toda prognosis política" (1965, p. 208). Precisamente, Freyer evita criticar esa tensión — semilla potencial de inconsistencias-, pues su trasfondo intelectual actuante es la misma "dialéctica de la historia", asumida virtualmente por él en varios de sus trabajos más tardíos. ${ }^{5}$

5 Por un lado, es interesante constatar cómo para el Freyer de los años sesenta del siglo xx, la aparición de los "sistemas secundarios", en el suelo abonado por el capitalismo industrial, ha terminado por apaciguar, "desde 
4.2. En las reflexiones metahistóricas de Friedrich Rapp se pueden encontrar ideas que emparientan su enfoque con el de Freyer. Una de ellas es la intuición central freyeriana de que el ser humano ha trazado y puesto en marcha, al modo de un "proyecto técnico", un prepotente y, en gran medida, impredecible "proceso objetivo". Con su ayuda, él también ha creado, con la forma de "sistemas secundarios" como los más nuevos medios de producción, transporte ¡y destrucción!, una especie de "segunda naturaleza" o "segundo Génesis" (Freyer 1965, p. 317). Ahora bien, la perspectiva de Rapp en modo alguno puede ser equiparada a la de un determinista "duro" o "romántico". En varios trabajos (e.g. 1978 y 1982) ha insistido en que del desarrollo tecnológico sólo puede derivarse un imperativo hipotético, abogar por un imperativo tecnológico implicaría sucumbir a la falacia naturalista (como lo habrían hecho tanto el movimiento tecnócrata como algunos enfoques cientificistas extremos). Pero su perspectiva tampoco calza con la perspectiva más influyente del antideterminismo constructivista y sociocéntrico. En la última década (e.g. 1992 y 1994), cabe percibir en sus escritos, como consecuencia tal vez de un mayor énfasis en el vasto contexto histórico del problema, una visión más favorable a ciertas

dentro del sistema", los ímpetus revolucionarios del marxismo decimonónico. El intercambio entre elementos deterministas y activistas se realiza ahora de una manera, hasta cierto punto, espontánea (como resultado, nuevamente, de un intrincado juego de "consecuencias no intencionadas" en las sociedades más industrializadas) o "no ideológica". Por otro lado, tal vez puede ser aún más interesante detectar ciertas afinidades estructurales entre el marxismo y la propia posición revolucionaria de derecha, impulsada por Freyer en diversos escritos de los años treinta y, en especial, en Freyer 1931. En esta obra, como Muller ha resaltado (p. 201), Freyer aparece como una especie de contrapartida conservadora-derechista de Lukács, en la medida en que apoya, como salida para el letargo y embobamiento cultural, un decidido activismo o voluntarismo político de parte del Volk alemán. Luego de la desastrosa experiencia nacionalsocialista, no es extraño que Freyer buscara con sus propios medios teóricos, como tantos otros intelectuales alemanes de derecha, dar cuenta de la nueva situación socio-política de posguerra. Sólo que en su caso, ni siquiera sus últimas obras se permiten excluir del todo cierta cuota de voluntarismo político (¡aunque, desde luego, sí se excluye del todo la figura del Führer!). Su eliminación definitiva habría resultado poco coherente con su propia visión tardía (pero ahora no autoritaria, sino liberal) de una historia abierta al futuro y a los cambios que dentro de ciertos límites puedan aún darse dentro de ella. Véase también, sobre este punto, la siguiente nota. 
preocupaciones del determinismo "duro". De su personal enfoque filosófico, en todo caso más bien atípico en la filosofía contemporánea de la tecnología, podría decirse que se ubica, mutatis mutandis, en la misma tradición intelectual de Löwith y Freyer, tanto en lo que se refiere a la aceptación de la "dialéctica de la historia", como en su fuerte tendencia hacia la elaboración de síntesis de amplio alcance histórico. Es entonces dentro de dicho marco, sensible a la evolución histórica tan particular de Occidente y su obsesión por el progreso y la factibilidad técnica, donde hay que situar el punto de vista de Rapp acerca del determinismo tecnológico.

Uno de los objetivos centrales del enfoque de Rapp consiste en llamar la atención sobre el hecho de que el actual proceso expansivo de tecnificación representa un caso más — no por ello completamente insólito - del determinismo propio de otros procesos típicos de la herencia occidental (europea moderna). Se trata de procesos como la secularización, la Ilustración, la democratización, así como diversos movimientos en procura de la emancipación política y la igualdad de oportunidades. Interesa destacar que en dichos acontecimientos actúa exclusivamente, para Rapp, un determinismo relativo, como corresponde a "fenómenos históricos, contingentes". Ninguno de ellos representa "dimensiones fijadas naturalmente", ni "son expresión de una necesidad esencial histórica" (1992, p. 103). El anterior es un punto de particular relevancia para Rapp; así, en su (1981) ya insistía vehementemente en que, al contrario de enfoques deterministas naturalizados, como los de S. Moscovici y S. Lem, el progreso tecnológico no puede considerarse "naturalmente", sino social y, por ende, normativamente determinado. Si bien podrían revelarse algunas similitudes externas entre las evoluciones biológica y técnica, el aspecto crucial de la presencia de valores y preferencias culturales en el progreso técnico deja atrás cualquier cosa parecida en la naturaleza y su discurrir "mecánico". El conjunto de las muy generales analogías de carácter fáctico con la evolución natural, "de ninguna forma representa una legitimación normativa para el ensanchamiento permanente de posibilidades de acción técnica" (p. 159). 
Contemplado desde una amplia perspectiva histórica cultural, el desarrollo tecnológico aparece en efecto, asegura Rapp, "como un acontecimiento global y suprapersonal, como un proceso histórico en el que la humanidad es al mismo tiempo sujeto y objeto" (1994, p. 148). El determinismo tecnológico no existe ciertamente como una fuerza personal conductora de la historia, la tecnología es obviamente de factura ciento por ciento humana. $\mathrm{Y}$, sin embargo, hay que tomar en cuenta "una experiencia fundamental de la historia", que consiste en "la diferencia entre intención y resultado". Se trata en suma de una "dialéctica de la historia", responsable de que, a partir de intereses y actos individuales, dirigidos a la realización de ciertas metas específicas a corto plazo, surjan finalmente, con el transcurrir histórico, "resultados finales de largo plazo, que no fueron deseados por los sujetos actuantes y que incluso se hallarían frecuentemente del todo fuera de su horizonte" (Rapp 1994, p. 129). Aunque esa "dialéctica" está presente en otros procesos históricos, Rapp aduce que tiende a ser más llamativa en el caso del actual desarrollo científico-tecnológico. Pero, con todo y todo, lo anterior no cierra la posibilidad y necesidad de ponderar y realizar otras opciones tecnológicas, menos ominosas que algunas de las actualmente en vigencia. Coincidiendo con la exigencia de Hans Jonas por una responsabilidad a largo plazo, respecto de las futuras generaciones, Rapp ha planteado con claridad en qué condiciones han de materializarse del todo ciertas aspiraciones o demandas éticas:

Cada uno de nosotros debe encarar la "dialéctica de la historia", es decir, el hecho de que, por un lado, las cosas sucederán, sin tener en cuenta lo que haga una persona individual, y, por otro, estamos obligados a considerar cuidadosamente lo que vayamos a hacer y a considerarnos responsables por los productos que surjan de nuestras acciones. (1991, p. 239)

\section{5. ¿Determinismo tecnológico o determinismo histórico?:} conclusiones y perspectivas

Es desde luego válido, pero también banal, afirmar que no existe algo así como la entidad "Tecnología" en tanto que figura regen- 
te del discurrir histórico. Ése es el corolario tan poco interesante a que conduce una concentración excesiva en el aspecto puramente instrumental de la actividad tecnológica. Por eso es más fecundo y estimulante adentrarse, para la reflexión filosófica, en la dimensión histórica, típicamente occidental en sus inicios, que está en juego en las nociones de "desarrollo" y "determinismo tecnológico". En tal sentido, es casi inevitable recurrir a una comparación de esas expresiones con la que fuera en otros tiempos la palabra casi mágica, de origen mayormente occidental, "progreso". Las tres cargan de modo inescapable con un contenido ambivalente: tanto en lo que se refiere a ese dinamismo optimista, característico de Occidente, dirigido a la conquista y transformación del mundo físico, como en lo que atañe al pesimismo por los efectos indeseados de dicha empresa expansiva. Sucede que existe, en el concepto de desarrollo tecnológico, la misma tensión que Freyer detecta en la noción más amplia y moderna, por lo menos desde la Ilustración, de progreso: "la tensión entre la idea de un avance progresivo conforme a la naturaleza y la idea de una realización activa por el hombre en el sentido del logro de una meta racionalmente conocida" (Freyer 1966, p. 220).

Una comprensión cabal de lo que está en juego con los términos de "progreso" y "determinismo tecnológico" exige reconocer, por ende, la influencia más general de la "dialéctica de la historia". Es que, al final, lo que se busca con aquellos dos conceptos es dar cuenta de situaciones complejas, en las que el grado de control racional implicado será siempre imperfecto y precario, situaciones en las que pareciera - dentro de un marco más global de determinismo histórico- que el determinismo tecnológico no resulta dispensable. Con ello no se está favoreciendo el fatalismo quietista ni la resignación pasiva (como aún se argumentará más adelante), sino simplemente se indican, de modo sobrio y objetivo, cuáles son, en el actual momento histórico, las reglas del juego. Porque, después de todo, como ha escrito Freyer, la "libertad sólo puede conservarse en situaciones presentes, en conexiones objetivas encontradas y en condiciones sociales dadas" (1965, p. 329). Todavía retomando una imagen trillada pero casi siempre eficaz, podría decirse que, en 
la medalla de la "dialéctica de la historia", la cara del progreso apunta hacia el aspecto más — pero no enteramente - voluntarista, indeterminado o abierto de la acción humana en la historia. Mientras que la cara del determinismo tecnológico lo hace hacia el aspecto más - pero no completamente - pasivo de dicha acción, aquel en que la complejidad del "sistema secundario de la cultura industrial" (Freyer) parece haber adquirido su propia "coacción objetiva" de desarrollo. El reto filosófico por asumir a continuación podría plantearse de la siguiente manera: aceptado el marco teórico propuesto en este trabajo, con la noción de la "dialéctica de la historia" en primer plano, ¿cómo sería posible conciliar, entonces, en una práctica socialmente eficaz, pero curada de optimismos ingenuos, ambas caras o facetas ineludibles en la relación del ser humano con la tecnología, una relación en la que éste aparece, por momentos, más como sujeto, pero en otros más como objeto de sus propias creaciones? Aunque no es posible ofrecer en este lugar una respuesta pormenorizada o completa a semejante interrogante, sí es necesario proporcionar al menos una indicación de la actitud crítica general que debería orientarla. Interesa, especialmente, matizar o hacer más precisa la alusión al elemento de pasividad vinculado con el progreso tecnológico.

La conformación de una actitud adecuada respecto del desafío determinista tecnológico, en tanto que manifestación de lo que ha sido establecido (desde las contribuciones de varios autores) como la "dialéctica de la historia", debe tomar en cuenta, en primer lugar, una serie de situaciones destacadas en el transcurso de este trabajo. Ante todo, se ha instado a reconocer la presencia actuante de cierto tipo de determinismo tecnológico resultado de la propia "dialéctica de la historia". Pero no se trata de un determinismo "duro", equiparable a una suerte de proceso natural, como el representado por la evolución biológica que se da en forma tan espontánea como repetitiva (no obstante la cada vez mayor intervención humana sobre sus mecanismos reproductivos). En este sentido, como ya se recalcó a propósito de los comentarios de Rapp sobre el tema, toda analogía entre la evolución natural y el progreso técnico debe verse con 
cierta sospecha. La "naturalización” completa del progreso tecnológico es una idea que debe rechazarse, pues resulta incompatible con una realidad de rasgos más complejos, que se capta mejor atendiendo a su carácter "dialéctico" (en el sentido ya comentado). En segundo término, se ha insistido en que una de las consecuencias de la atención prestada al fenómeno del determinismo tecnológico —como un elemento más de la amplia gama de acontecimientos históricos interactuantes - podría ser, en efecto, cierto sentimiento pesimista de indefensión, de verse desalentadoramente abrumado por "la dinámica interna del coloso técnico" (H. Jonas) y su ilimitado poder coactivo. Ésa no es, sin embargo, ni mucho menos, toda la verdad: la consideración de lo que aparenta ser un discurrir histórico (y en su interior el propio desarrollo tecnológico) tal que, "dirigiéndose" a metas inciertas, pareciera a veces que lo arrastra todo consigo, y ante el cual parece inútil ofrecer resistencia, no debería constituir por sí misma una ocasión para la resignación ante una tecnología autónoma. Antes bien, dicha consideración podría constituirse en oportunidad para columbrar de un modo sobrio, objetivo y "realista" las condiciones históricas que hay que tomar en cuenta para todo intento de cambio o reforma. Se trata de condiciones que determinan notablemente, pero sólo hasta cierto punto, la conformación de caminos futuros para una mejor calidad de vida.

Precisamente, en esta coyuntura resulta apropiada una mención final a Hans Jonas. Este autor, conocido hoy sobre todo por sus trabajos en la bioética, tuvo la suficiente perspicacia como para reconocer la complejidad de la situación actual de Occidente, marcada por la omnipresencia de la tecnología, y para sugerir a la vez una serie de ideas encauzadas a combatir el fatalismo absoluto, la rendición incondicional ante lo que él consideró uno de los rasgos centrales del "síndrome tecnológico que tiene una importancia ética propia: el elemento cuasiforzoso de su avance, que por así decirlo hipostatiza nuestras propias formas de poder en una especie de fuerza autónoma de la que nosotros, los que la ejercemos, nos volvemos paradójicamente súbditos" (1997, p. 38). La importancia de ciertas intuiciones de Jonas para la perspectiva final que el presente trabajo desea sugerir, o al menos dejar abierta, estriba en que desde su enfoque se reconoce 
y acepta en toda su magnitud el determinismo tecnológico — en el sentido aquí delimitado como parte de la "dialéctica de la historia"-, pero, al mismo tiempo, se alienta e incluso se exige configurar cierta actitud por parte de los sujetos humanos, que procure apartarse lo más posible de la aciaga idea de que puesto que no se puede cambiar la Summa tecnológica, ergo no se puede cambiar nada en particular. En este sentido se distingue la convergencia de autores con preocupaciones tan diversas como Freyer, Rapp y Jonas. Sus críticas independientes se enfilan a tendencias políticas utópicas o quiliásticas, con su proclividad a visualizar una especie de "cierre" definitivo de la historia, y de la posibilidad de modificar el modo en que se encarrilan ciertos desarrollos tecnológicos. ${ }^{6}$ Una vez abierta, como diría Jonas, la caja de Pandora de la tecnología, y sabiendo que no queda más remedio "que vivir con la ambigüedad de todo lo humano" (1997, pp. 90 y 41-54), no queda otra alternativa viable más que reforzar socialmente tanto ciertos valores "anticuados" - la moderación, la humildad y la austeridad - así como el papel de las instancias e instituciones encargadas de vigilar que los avances tecnológicos no se conviertan en determinantes absolutos de la acción humana.

\section{BIBLIOGRAFÍA}

Basalla, George, 1988, The Evolution of Technology, Cambridge University Press, Cambridge y Nueva York.

6 Ya se indicaron las reservas de Freyer frente al quiliasmo de ascendencia marxista. Sin embargo, para reforzar aún más lo que acaba de comentarse, atiéndase el siguiente texto de Freyer, que también Jonas podría suscribir perfectamente: "Sólo la ficción del quiliasmo cierra la historia con un último fin allende el cual no hay futuro. La verdadera historia está siempre abierta por su cara anterior y, aun cuando la enajenación amenaza cerrarla como callejón sin salida, nunca debemos decir que jamás hubiere de continuar" (1966, pp. 258259). A la par de enérgicas son las opiniones jonasianas en contra del utopismo marxista - en especial el propugnado por Ernst Bloch. Jonas también se resiste a creer que la historia posea una suerte de "automovimiento necesario, con su meta implantada y quizás hasta reconocible, contra la que sería vana toda resistencia". Cfr. su obra central, 1995, p. 355. 
Bimber, Bruce, 1996, "Tres caras del determinismo tecnológico", en Merritt Roe Smith y Leo Marx (comps.), 1996, pp. 95-116.

Ellul, Jacques, 1954, La Technique ou l'enjeu du siècle, A. Colin, París.

Ferguson, Adam, 1995, An Essay on the History of Civil Society, Cambridge University Press, Cambridge y Nueva York.

Freyer, Hans, 1966, Teoría de la época actual, 2a. ed., Fondo de Cultura Económica, México.

—— 1965, Schwelle der Zeiten. Beiträge zur Soziologie der Kultur, Deutsche Verlags-Anstalt, Stuttgart.

- , 1931, Revolution vom rechts, Diederichs, Jena.

Glaser, Wilhelm, 1972, Soziales und instrumentales Handeln. Probleme der Technologie bei Arnold Gehlen und Jürgen Habermas, Kohlhammer, Stuttgart.

Hegel, G.W.F., 1999, Lecciones sobre la filosofía de la historia universal, trad. José Gaos, Alianza, Madrid.

Huisinga, Richard, 1996, Theorien und gesellschaftliche Praxis technischer Entwicklung. Soziale Verschränkungen in modernen Technisierungsprozessen, G+B Verlag Fakultas, Amsterdam.

Jakob, Eric, 1996, Martin Heidegger und Hans Jonas. Die Metaphysik der Subjektivität und die Krise der technologischen Zivilisation, Francke, Tubinga y Basilea.

Jonas, Hans, 1997, Técnica, medicina y ética. Sobre la práctica del principio de responsabilidad, Paidós, Barcelona.

- , 1995, El principio de responsabilidad. Ensayo de una ética para la civilización tecnológica, Herder, Barcelona.

—_, 1966, "Gnosticism, Existentialism, and Nihilism", The Phenomenon of Life. Towards a Philosophical Biology, Harper \& Row, Nueva York.

Jünger, Ernst, 1932, Der Arbeiter: Herrschaft und Gestalt, Hanseatische Verl.-Anstalt, Hamburgo.

—_ y Martin Heidegger, 1994, Acerca del nihilismo, Paidós, Barcelona.

Kant, Immanuel, 1987, Ideas para una historia universal en clave cosmopolita y otros escritos sobre filosofía de la historia, Tecnos, Madrid.

Leiss, William, 1990, Under Technology's Thumb, McGill-Queen's University Press, Montreal y Kingston.

Löwith, Karl, 1983, Weltgeschichte und Heilgeschehen. Zur Kritik der Geschichtsphilosophie. Sämtliche Schriften, vol. 2, Metzler, Stuttgart.

—_, 1963, “Das Verhängnis des Fortschritts”, en 1983, pp. 392-410. 
__ 1953, Heidegger —Denker in dürftiger Zeit, S. Fischer, Francfort del Meno.

—_, 1950, "Natur und Geschichte", en 1983, pp. 280-295.

_ 1949, Meaning in History. The Theological Implications of the Philosophy of History, The University of Chicago Press, Chicago.

Muller, Jerry Z., 1987, The Other God that Failed: Hans Freyer and the Deradicalization of German Conservatism, Princeton University Press, Princeton, N.J.

Niiniluoto, Ilkka, 1990, "Should Technological Imperatives Be Obeyed?", International Studies in the Philosophy of Science, vol. 4, no. 2, pp. 181-189.

Rapp, Friedrich, 1994, Die Dynamik der modernen Welt. Eine Einführung in die Technikphilosophie, Junius, Hamburgo.

—_, 1992, Fortschritt. Entwicklung und Sinngehalt einer philosophischen Idee, Wiss. Buchges, Darmstadt.

— , 1991, "Responsibility Allocation in Modern Technology", en J. Rasmussen et al. (comps.), Distributed Decision Making, Wiley, Chichester, pp. 233-246.

—_, 1982, "Philosophy of Technology", en G. Flöistad (comp.), Contemporary Philosophy: A New Survey, vol. 2, Nijhoff, La Haya, pp. 361-412.

_, 1981 , “Die Technik als Fortsetzung der Evolution?”, en F. Rapp (comp.), Naturverständnis und Naturbeherrschung, Fink, Munich, pp. 145-160.

—_, 1978, Analytische Technikphilosophie, Alber, Friburgo y Munich.

Ropohl, Günter, 1983, “A Critique of Technological Determinism”, en Paul T. Durbin y F. Rapp (comps.), Philosophy and Technology, Reidel, Dordrecht, pp. 83-96.

Rosales, Amán, 1994, Die Technikdeutung Martin Heideggers in ihrer systematischen Entwicklung und philosophischen Aufnahme, Projekt Verlag, Dortmund.

Smith, Merritt Roe, y Leo Marx (comps.), 1996, "Introducción”, en Historia y determinismo tecnológico, Alianza, Madrid, pp. 11-17.

Sullivan, John E., 1970, Prophets of the West. An Introduction to the Philosophy of History, Holt, Rinehart y Winston, Nueva York.

Teusch, Ulrich, 1993, Freiheit und Sachzwang. Untersuchungen zum Verhältnis von Technik, Gesellschaft und Politik, Baden-Baden, Nomos.

Van der Pot, Johan Hendrik, 1985, Die Bewertung des technischen Fortschritts. Eine systematische bersicht der Theorien, Van Gorcum, Assen. 
Winner, Langdon, 1977, Autonomous Technology. Technics-out-ofControl as a Theme in Political Thought, The MIT Press, Cambridge, Mass., y Londres.

Recibido el 26 de enero de 2001; revisado el 12 de septiembre de 2001; aceptado el 7 de noviembre de 2001 